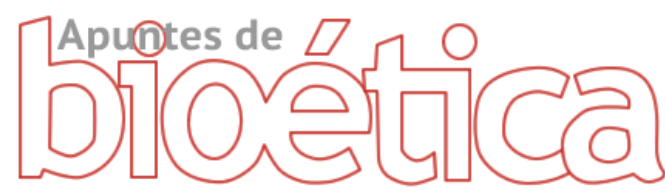

https://doi.org/10.35383/apuntes.v2i2.291

\title{
Criterios teóricos y prácticos que orientan el uso de dispositivos móviles en la comunicación familiar con adolescentes
}

\author{
Janett Melita Gómez Ibañez ${ }^{1}$
}

INFORMACIÓN DEL ARTÍCULO RESUMEN

\section{Historia del artículo:}

Recibido el 26 de setiembre de 2019

Aceptado el 22 de diciembre de 2019

\section{Palabras claves:}

Comunicación familiar

Dispositivos móviles

Adolescentes

En la base del contexto actual de uso de dispositivos móviles por parte de adolescentes, surge la necesidad de establecer los criterios teóricos y prácticos para promover la comunicación familiar partiendo de esta realidad. Desde un estudio cuanti - cualitativo, descriptivo e interpretativo, se ha identificado teorías que apoyan la inserción de estos dispositivos, así como las que califican su uso como objetos de conflicto e intrusión familiar. De este modo, se logró clasificar su utilidad y funcionalidad así como el abuso y dependencia. Se concluyó que, en la etapa temprana adolescente existe una moderada extensión de uso que se incrementa progresivamente con la edad, y la necesidad del diseño de propuestas que orienten a las familias acerca de las ventajas y riesgos que el uso de estos dispositivos generan.

Theoretical and Practical Criteria Orienting the use of Mobile Devices in Family Communication with Teens

\section{ABSTRACT}

Keywords:

Family communication

mobile devices

adolescents
On the basis of the current context of use of mobile devices by adolescents, the need arises to establish the theoretical and practical criteria to promote family communication based on this reality. From a quantitative, descriptive and interpretive study, theories were identified that support the insertion of these devices as well as those that describe their use as objects of conflict and family intrusion.

It was concluded that, in the early adolescent stage there is a moderate extension of use that increases progressively with age, and the need to design proposals that guide families about the advantages and risks that the use of these devices generate.

1 Magister de Persona, Matrimonio y Familia, asesora y conferencista en comunicación familiar y relaciones humanas en instituciones educativas. Email janettmelita@gmail.com. ORCID: https://orcid.org/0000-0002-5964-3532 


\section{Introducción}

Actualmente, de acuerdo a la posición del autor Riesco (2014), los Dispositivos Móviles son las herramientas tecnológicas perfectas que ayudan a perpetuar el crecimiento y desarrollo de las nuevas generaciones así como el de generaciones anteriores que, sorprendentemente también se encuentran inmersas en estos procesos, creando múltiples alternativas y beneficios, produciendo, comentando o compartiendo (p. 90).

Las estadisticas demuestran que se han superado los miles de millones de dispositivos móviles en el mundo ${ }^{2}$ y los valores se incrementan por la insaciable necesidad de estar siempre conectado.

Sin embargo, investigaciones recientes han cuestionado mucho si este avance avasallante de dispositivos y la poca normatividad que existe en su uso, está afectando de modo negativo el desarrollo de las relaciones humanas, la educación y por ende a la familia. Esto invita a reflexionar como en tan corto tiempo, los patrones tradicionales de comunicación se han ido modificando, revelando en muchos casos, situaciones que alteran las conductas en los niños y adolescentes, poniendo en riesgo su desarrollo emocional, sus derechos y hasta su propia vida.

Con el propósito de dar nuevas propuestas que ayuden a buscar una solución, se planteó la necesidad de investigar cuáles son los criterios que deben ser tenidos en cuenta por las familias para orientar el uso de estos dispositivos móviles en la comunicación con adolescentes, y a partir de este planteamiento, se buscó trabajar el estudio con un centro educativo del distrito de San Miguel en la ciudad de Lima. El director, la

(2) Estudios realizados en Europa, Asia y América Latina, revelan las tendencias del uso local que indica el incremento. Redacción gestión. (4 de julio de 2015) El lado oscuro de la tecnología. GESTION. Recuperado de http://gestion.pe/tecnologia/lado-oscuro-tecnologia-2136292. plana docente como los padres de familia, pusieron de manifiesto una preocupación acrecentada por el desafío de afrontar estos nuevos retos. Es así que se propuso el estudio de estos estilos de comunicación, llámese lenguaje digital por dispositivos móviles, en alumnos de etapa adolescente temprana de $1^{\circ}$ año de secundaria, en edades que oscilan entre los 11 a 13 años.

Para ello, se elaboró una encuesta dirigida a los alumnos del plantel, a fin de tener la información necesaria sobre la percepción de los adolescentes y la de sus progenitores en cuanto al nivel de acogida de esta tecnología, así como también, recopilar información de investigaciones científicas sobre contenidos de la comunicación, la familia y los dispositivos tecnológicos, planteando las respectivas variables

De este modo, se determinó como objetivo general, establecer los criterios teóricos y prácticos que deben orientar el uso adecuado de los Dispositivos Móviles, caracterizando el contexto en el marco de la comunicación de las familias modernas, analizando los aspectos positivos y negativos que ayuden a determinar criterios que deban ser tenidos en cuenta por los padres y docentes.

\section{Metodología}

El estudio, se desarrolló desde una base teórica, partiendo desde dos premisas que se manifiestan en las familias actuales: la intrusión de los dispositivos en el hogar y el modo en que los padres manejan la comunicación con sus hijos adolescentes desde diferentes estilos educativos de crianza.

Para lograr los resultados esperados, el estudio partió desde un enfoque de tipo cuanti- 
cualitativo, descriptivo e interpretativo, correlacional, que permitió analizar las bases teóricas sobre los estilos de crianza parentales, los tipos de comunicación abierta y cerrada que se producen en la interacción dentro de la comunicación familiar, así como la comunicación verbal, no verbal y digital; de este modo, la investigación permitió paralelamente conocer los avances tecnológicos en cuanto a uso y características en el campo de las tecnologías de la información y comunicación.

Tanto el marco teórico expuesto como los antecedentes encontrados, fueron trabajados con fichas de resumen analítico estructurado (RAE de 1-49), los cuales fueron corroborados con datos realizados a través de la encuesta aplicada a los estudiantes ${ }^{3}$, y el apoyo de aportes de observación a las familias que acudieron a las charlas de la escuela de padres en la institución educativa.

La encuesta aplicada se desarrolló de acuerdo a las variables de estudio: adolescentes, dispositivos móviles y comunicación familiar, fue estructurada en secciones o tablas de preguntas, tales como: los tipos de comunicación abierta o cerrada; formas de comunicación verbal y no verbal, el uso de los estilos educativos parentales en la crianza; la problemática en la comunicación familiar, los factores de influencia negativas y positivas de los dispositivos en el hogar; factores de entretenimiento; factor social; factor educativo y factores de riesgo de uso.

Una vez aprobada la encuesta planteada, tanto por psicólogos especialistas, como por el director y el departamento psicológico del plantel, se procedió a aplicar dicha encuesta a los sujetos de investigación, que fueron 58 alumnos de ambos sexos, 27 de sexo femenino y 31 de sexo masculino, todos de etapa temprana

(3) Tomando en cuenta que en el 2015 se inició la investigación así como la aplicación de la encuesta a los alumnos que pertenecían al nivel $1^{\circ}$ de secundaria en ese entonces, el planteamiento del análisis se realizó a comienzos del año escolar de 2016.
(11-13 años de edad). Los resultados encontrados se procesaron y evaluaron según la escala de Likert, identificándolos en las tablas trabajadas. Utilizando el software SPSS 20, Para confiabilidad del instrumento se validó de forma cuantitativa, una prueba piloto aplicada a estudiantes de otra aula con las mismas características de la muestra de estudio.

\section{Análisis}

Al tener listos los resultados de la encuesta, estos se pudieron dividir en cinco apartados que ayudaron a desarrollar los objetivos planteados:

\section{La Caracterización de la familia actual,}

este primer apartado analizó las implicancias que generan el estilo de crianza y el uso de los dispositivos móviles en el hogar. Los adolescentes crean su propio espacio de conexión virtual con sus iguales, generando en la relación con los padres, barreras impenetrables de comunicación (Tueros, 2002, p. 23). Esta barrera vista desde un corte psicológicosocial y sistémico ${ }^{4}$, describe los resultados exponiendo los tipos de comunicación que existe en la relación parental sea abierta o cerrada, las formas del lenguaje, la expresión, así como la conexión digital adolescente.

Cabe señalar que los resultados presentaron marcados estilos de crianza tanto en el desarrollo educativo como en el social, revelando en algunos casos padres con una tolerancia excesiva frente a la conducta del adolescente, y otros una restricción y control riguroso en el desarrollo de sus hijos.

\footnotetext{
(4) El flujo comunicativo en las familias y su desarrollo, se establece de acuerdo a los mensajes positivos (abiertos) o negativos (cerrados) que comuniquen; de modo que el aprendizaje constante de soluciones o conflictos que se produzcan, marcarán las interacciones a lo largo de su desarrollo y relaciones futuras, adecuándose a los medios que necesiten. (Satir, 2002, p. 45).
} 
Por otro lado, aunque no muy marcado, se encuentra una crianza negligente carente de control y orientación que vigile y eduque las conductas de los mismos, como así sucede en un estilo democrático de crianza, que posee un nivel de control adecuado sobre el adolescente y permite independencia de sus actos con la debida orientación de sus padres.

En los resultados, se observó que los tipos de comunicación que los adolescentes mantienen en el hogar, se establecen con una comunicación abierta en familias democráticas, cerrada en familias restrictivas o permisivas y en ambas el uso de la comunicación digital se manifiesta como una necesidad imperante de conexión pero con diferentes fluctuaciones.

La presente tabla muestra cómo se manifiesta la comunicación, sea de tipo abierta o cerrada y la relación existente entre los estilos educativos parentales.

\begin{tabular}{|c|c|c|c|c|c|c|c|c|}
\hline \multirow[b]{2}{*}{ condición } & \multicolumn{2}{|c|}{$\begin{array}{l}\text { OMUNICACIÓN } \\
\text { LUIDA/ABIERTA }\end{array}$} & \multicolumn{2}{|c|}{$\begin{array}{c}\text { APOYO } \\
\text { SOLUCIÓN DE } \\
\text { PROBLEMAS }\end{array}$} & \multicolumn{2}{|c|}{$\begin{array}{l}\text { COMUNICACIÓN } \\
\text { CERRADA }\end{array}$} & \multicolumn{2}{|c|}{$\begin{array}{c}\text { NO HAY } \\
\text { INTERACCIÓN }\end{array}$} \\
\hline & $\mathrm{n}^{\circ}$ & $\%$ & $n^{\circ}$ & $\%$ & $\mathrm{n}^{\circ}$ & $\%$ & $n^{\circ}$ & $\%$ \\
\hline $\begin{array}{l}\mathrm{Si} \\
\text { presenta }\end{array}$ & 45 & $78 \%$ & 37 & $64 \%$ & 17 & $29 \%$ & 5 & $9 \%$ \\
\hline $\begin{array}{l}\text { No } \\
\text { presenta }\end{array}$ & 11 & $19 \%$ & 21 & $36 \%$ & 41 & $71 \%$ & 53 & $91 \%$ \\
\hline total & 56 & $97 \%$ & 58 & $100 \%$ & 58 & $100 \%$ & 58 & $100 \%$ \\
\hline
\end{tabular}

Estos indicadores muestran una interacción familiar adecuada, sin embargo existe un porcentaje de encuestados que presentan problemas de comunicación en el hogar.

\section{Lenguaje y Expresión vs. Conexión digital}

Tomando como referencia el enfoque sistémico de la comunicación familiar (Satir,
2002, P. 95), la función principal de ésta es lograr que el mensaje llegue adecuadamente al receptor, para lo cual se valdrá de todos los medios para expresar lo que desea comunicar. Según el estilo educativo familiar que adopten los padres, éstos podrán o no adaptarse a los cambios que presenten los adolescentes con sus rasgos físicos y psicológicos propios de la edad,... "siendo apoyo y orientación en las nuevas formas comunicativas que intervengan en la relación"... (Antolinez, Rivero y Torres, 2011), y así como también en la calidad de tiempo que les brinden.

Se muestra que existe una tendencia a una comunicación no verbal inadecuada, que se pone de manifiesto en las conductas que toman los adolescentes, siendo propio de la edad; sus manifestaciones sociales con sus pares y aspectos psicológicos de independencia y aceptación de sí mismo.

La presente tabla muestra las formas comunicativas; lenguaje y expresión que manifiestan de forma adecuada o inadecuada:

\begin{tabular}{lcc|cc}
\hline FORMAS & \multicolumn{2}{c}{$\begin{array}{c}\text { COMUNICACIÓN } \\
\text { VERBAL }\end{array}$} & \multicolumn{2}{c}{$\begin{array}{c}\text { COMUNICACIÓN NO } \\
\text { VERBAL }\end{array}$} \\
\cline { 2 - 5 } & $\mathrm{n}^{\circ}$ & $\%$ & $\mathrm{n}^{\circ}$ & $\%$ \\
\hline $\begin{array}{l}\text { Com. } \\
\text { Adecuada }\end{array}$ & 49 & $74 \%$ & 27 & $29 \%$ \\
\hline $\begin{array}{l}\text { Com. } \\
\text { Inadecuada }\end{array}$ & 9 & $26 \%$ & 31 & $71 \%$ \\
\hline total & 58 & $100 \%$ & 58 & $100 \%$ \\
\hline
\end{tabular}

La tabla presenta a un número de encuestados que afirmó que el diálogo con sus padres es fluido, libre y se complementa con la comunicación no verbal de manera apropiada, un sector de los encuestados confirma que al expresar sentimientos o dudas, son escuchados (Satir, 2002, p. 97). Por el contrario, en otro sector disminuye la comunicación verbal en aquellos que 
presentan conflictos con sus padres, frente a un sector mayoritario que manifiesta una adecuada comunicación no verbal. Esto advierte que las formas de expresión, como comunicarse con calor afectivo, por contacto o gestos, puede estar perdiendo fuerza con el tiempo y el incremento de la edad (Jiménez T. 2006. p. 125), y dando paso a formas menos manifiestas y más abstractas de demostrar afecto. Esta dificultad que manifiestan en la comunicación no verbal se revela aún más, según Satir, en el método de adivinar el pensamiento (Satir, 2002, p. 87).

Los padres al estar preocupados por la manera en la que se comunican hoy sus hijos, suelen muchas veces manifestar sus emociones sobredimensionando sus gestos y acciones, lo que genera en ellos un mensaje distorsionado que dificulta la comunicación, creando rechazo y confusión en quienes atraviesan una etapa en la cual empiezan a sociabilizar (Satir, 2002, p. 396).

Estas trampas de la comunicación afectan la manera de relacionarse y expresar satisfactoriamente sus pensamientos, modifican el desarrollo natural de su valía y en consecuencia la interacción con sus padres.

Los resultados evidencian que las formas de comunicación (verbal y no verbal) se complementan entre sí, resultando en la emisión del mensaje completo y que puede o no manifestarse a través de patrones, que resuelven o dificultan la vida diaria de las personas mediante distintas reacciones $y$ efectos, sean estos negativos como el estrés o la tensión, o positivos, con expresiones de afecto y sosiego ${ }^{5}$. "Una buena comunicación con ambos padres (abierta, afectiva y satisfactoria) favorece la autoevaluación positiva en el adolescente en todas las áreas de su autoestima" (Jiménez T. 2014, p. 96).

Por otro lado, la encuesta refleja que la conexión digital en la percepción adolescente; complementa el diálogo como una forma de expresión en sus estados, lo que para el padre de familia, en muchos casos no considera adecuado y manifiesta su descontento.

Características de uso de los DM en el hogar, Los antecedentes encontrados de investigaciones realizadas recientemente sobre este tema, han evidenciado en el presente análisis que tanto los tipos y formas de comunicación entre adolescentes y sus padres, presentan aspectos que han generado una creciente tensión en las relaciones familiares, así lo demuestran los datos recopilados tanto en la encuesta como en la observación realizada en las charlas de Escuela de Padres en el plantel.

- Una de las características más relevantes en los encuestados, es que la mayoría considera la necesidad imperiosa de estar conectados a sus aplicaciones ininterrumpida y simultáneamente a través de sus dispositivos, pues consideran la comunicación digital una herramienta indispensable para relacionarse, debido a que todo su mundo social está en el dispositivo. Las respuestas resultan cuestionables, si se hace referencia a indicadores como la influencia del dispositivo, por el cual los encuestados valoran la necesidad del mismo como una herramienta que facilita el diálogo, aun cuando el caso sea comunicarse con sus padres

(5) Satir, Patrones de Comunicación, p. 94. 
por emergencia o localización, dentro del cual, denota que hay una norma basada en la preocupación por el otro (Jiménez, 2015, p. 10); se deduce entonces que existe una leve influencia de los DM en los encuestados sin que esto llegue a generar algún tipo de necesidad arraigada, pero que puede acrecentarse con los medios de consumo masivo que están amalgamando esa necesidad.

- El apoyo que los padres atribuyen a los DM como herramientas útiles de control y monitoreo, (Medina, 2013, p.6), considerando una herramienta válida de apoyo en tiempos de inseguridad, permitiendo el monitoreo cuando ellos están fuera de casa.

$>$ El cordón umbilical electrónico o correa digitap. Es el vínculo que se establece entre los miembros de una conexión digital, visto desde la perspectiva local, los encuestados se sienten más valorados y seguros.

La presión paterna de control e invasión de privacidad, que el adolescente percibe, al ser vulnerado en su espacio íntimo.

Las falsas interpretaciones crean trampas en la comunicación. Hay un sector importante en los indicadores de la encuesta que revela que las familias de hoy, suelen enviarse o recibir mensajes confusos, que no ayudan a una buena comprensión. Satir (2002), fundamenta que en las relaciones humanas esto se debe a que las personas llevamos una historia preconcebida de los demás de acuerdo a la primera impresión que se manifiesta y no dependen del objeto en discusión, sino de la actitud que asume cada miembro.

$>$ Los padres prefieren comunicarse de manera analógica (Herrero y Alcalde, 2014, p. 193). El manejo de las aplicaciones resulta más compleja para los inmigrantes digitales que para los nativos digitales. A un sector de los encuestados les parece lento y poco atractivo este tipo de comunicación, para ellos, las experiencias en el campo de la conexión, de padres y abuelos, no encajan en el mundo del adolescente, por lo que procuran mensajes de contenido abreviado y conectivo.

Restricción e indiferencia vs. Interés y aceptación, se ha identificado que la influencia de los estilos de crianza, dependiendo del estilo que los padres apliquen con sus hijos adolescentes, se acentuará en un clima de aceptación, rechazo o indiferencia al uso de las nuevas tecnologías portátiles. Desde la percepción adolescente, será adecuado, cuando exista un buen flujo de comunicación positiva entre ellos, o inadecuado en una comunicación conflictiva o nula.

\section{Ventajas que existen entre el uso de los dispositivos por adolescentes y la influencia del estilo de crianza}

(6) Ling, 2004. citado por Fidalgo et al 2013, refiriéndose a poner en contacto a las personas en cualquier momento, haciendo énfasis en la relación personal más que en una localización material. Jiménez L., 2014 p. 7. 
En este apartado se consideró las opiniones de los padres en las charlas de escuela de padres a conversaciones casuales sobre el uso de los DM, compartiendo experiencias con otros padres y la plana docente del colegio:

Fomenta una comunicación más cercana entre el trabajo y el hogar. Una de las ventajas más importantes que se ha podido observar en los DM, es que ha ido eliminando los límites de comunicación entre el trabajo y el hogar, lo cual se observa en los resultados de la encuesta en el cual la mayoría afirma que sus padres (poco permisivos o democráticos) se comunican con ellos a través de los chats, confirmando que la función básica de éstas tecnologías es mantener el contacto con sus hijos en su ausencia.

Se establece un hilo virtual, de comunicación y de control de los miembros en sus desplazamientos fuera del hogar (Antolinez, Rivero y Torres, 2011, p.20). Otra de las ventajas en el estilo democrático, es que los encuestados afirman que respetan las decisiones y la orientación que sus padres les brindan. En este sentido, es prudente afirmar que en la investigación que Jiménez hizo acerca de las prácticas de intimidad familiares, se pudo establecer que a través de una de las aplicaciones más usadas en los DM como es whatsapp, aunque los miembros se encuentren distantes el uno del otro, sienten la necesidad de expresar afecto a través de gráficos y texto, y se preocupan por su seguridad e integridad. Estos aspectos se identifican en los padres de familias monoparentales ${ }^{7} q u e$ pese a la ausencia de uno de ellos en la crianza, como padres orientadores y preocupados, tienen la necesidad de estar cerca de su familia y mantienen un estilo de diálogo abierto y democrático ${ }^{8}$.

> Facilita la estructuración de espacios y tiempos. Como lo denomina Ling, "micro coordinación", las herramientas que se manejan en los smartphones, tablets, o celulares comunes, sirven a las familias para estructurar el manejo y la organización de sus labores cotidianas. Eso ayuda a organizar horarios distribuyendo las actividades según las necesidades familiares.

$>$ Permite expresar afecto a través del chat, lo que es difícil decir cara a cara (Jiménez. L. 2014, p. 17). Las aplicaciones utilizadas en el DM complementan el contacto afectivo, mitigando la preocupación de los padres, y ello favorece la solución de desavenencias al apaciguar conflictos con los hijos, a través de caracteres que simbolizan emociones.

$>$ Produce perpetuidad de vivencias y recuerdos a través de las aplicaciones multimedia, genera acercamiento y unidad mediante el

\footnotetext{
(7) "La emergencia de las familias monoparentales, particularmente demandantes de lazos telefónicos personalizados en razón a su estructura "rota"." GARCÍA Y MONFERRER. Propuesta de análisis teórico sobre el uso de teléfonos móviles en adolescentes. Comunicar. № 33. Vol. XVII. 2009. P. 86. (8) Este resultado se pudo confirmar en la sesión de escuela de padres en que se discutieron los criterios que los padres tenían acerca de las ventajas y desventajas de la comunicación mediante los dispositivos móviles con sus hijos. Charla del 22 de mayo de 2016.

${ }^{(9)}$ Ling. 2004. (Citado por Jiménez L. 2014, p. 18).
} 
entretenimiento familiar, con fotos, videos y juegos en red, en el que tanto padres como hijos comparten un espacio de intimidad familiar ${ }^{10}$.

Produce una manifestación recíproca de ayuda entre los saberes del nativo digital y el inmigrante digital. Se crea una especie de acuerdo tácito de cooperación en que los jóvenes adiestran a sus padres o abuelos inmigrantes digitales ${ }^{11}$, en el uso de los DM y las aplicaciones para estar interconectados, esto favorece la comunicación rápida, sencilla con los padres, sobre todo con las madres, que están más pendientes de sus hijos, de modo que éstos a su vez enseñan a sus hijos el correcto uso de tiempo, distinguiendo con su orientación lo que es íntimo, lo que es privado y lo que es público.

Consideran que suple el vacío de la intimidad familiar en padres ausentes (García y Monferrer. 2009, p. 84). Se identificó un porcentaje de encuestados con padres indulgentes, que brindan todo material tecnológico para su educación y distracción. Los padres de este estilo de crianza (permisivos) consideran el DM un medio oportuno de cubrir el vacío por actividades que generan ausencias, cumpliendo con las exigencias que sus hijos reclamen. En este aspecto, el riesgo es mayor al caer en conductas adictivas de uso, sin orientación parental presente, así como a gastos abusivos con costos elevados, lo cual representa para la

\footnotetext{
(10) Charla Escuela de Padres. Comunicación Familiar. del 22 de mayo de 2016.

(11) Véase comunicación de los padres a través de los chats. El manejo de las aplicaciones es más complicada para los inmigrantes que para los nativos digitales. (Gómez. J. 20, 2016 p. 72).
}

sociedad de consumo grandes oportunidades de incremento del mercado digital.

\section{Desventajas que existen entre el uso de los dispositivos por adolescentes y la influencia del estilo de crianza:}

Respecto a las desventajas es propio indicar:

Existe una norma ambigua de restricción, que los padres imponen restringiendo los celulares, cuando estos invaden el horario de las comidas, sin embargo a veces, ellos mismos tienden a quebrar sus propias normas, creando un doble discurso.(Antolinez, Rivero y Torres, 2011, p. 12).

Uno de los conflictos más analizados, es el grado de confianza que se manifiesta de acuerdo al estilo de crianza parental que ejerza el progenitor sobre el adolescente, tomando en cuenta que la comunicación actual con sus hijos, dependerá también del grado de conocimiento que los padres tengan acerca de estos nuevos medios digitales y sus aplicaciones, así como conocer los intereses de sus hijos.

Consentimiento de intrusión en los espacios íntimos. La inmediatez y la rapidez son características que puede generar problemas en las aplicaciones, permitiendo a personas tímidas relacionarse más fácilmente a las redes sociales siendo receptores de todo tipo de contenidos (García y Monferrer, 2009, p. 85); pueden mentir acerca de sí mismos y compartir sus espacios con desconocidos. Se ha observado que en padres con un estilo de crianza ausente, permisiva, muy autoritaria y casos extremos negligente, con hijos en posesión de varios dispositivos móviles en casa 
durante todo el tiempo, pasa desapercibido el control de los mismos, quedando expuestos a páginas de contenido violento, sexual, ideológico $u$ otros tipos de consumo que trasgreden principios éticos y morales en la formación adolescente.

- Exagerada presión y alto nivel de expectativa en el comportamiento correcto del hijo, que con una comunicación pobre en la relación puede llevar a que los jóvenes cedan al abuso de poder sobre otros pares, tratan de expresar sus sentimientos $y$ emociones íntimamente a sus contactos de forma desmedida y sin control, generando un sub mundo en el hogar. En estudios encontrados de este estilo parental, realizados en países desarrollados ${ }^{12}$, hablan de la indiferencia y deshumanización que puede ocasionar este tipo de crianza en la identidad del hijo, creando comportamientos desmedidos de intimidación y autoestima baja. En este sentido, el whatsapp se ha convertido en la aplicación más usada desplazando a Facebook, por ser un medio más privado de comunicación con sus pares y evitar que los padres hurguen y controlen sus contenidos.

$>$ Crianza que no ostenta normas de comportamiento y control adecuado sobre el uso y el respeto a la moral en sus hijos (García y Monferrer, 2009, p. 85). Hay un sector de los encuestados que confirma este enunciado, al contar con total libertad de uso de sus dispositivos sin ser cuestionados por sus padres ante el abuso de los mismos. $Y$ en efecto, este sector de jóvenes puede manifestar conductas susceptibles de infringir no solo acoso escolar a otros mediante las redes sociales, sino compartir contenidos de índole sexual y violencia inapropiados para su edad con predominante poder $\mathrm{y}$ total indiferencia a los valores éticos y morales.

$>$ Total desapego e indiferencia en la crianza ausente de comunicación. Este fenómeno se puede apreciar en un estilo de crianza negligente, en el cual no existe relación afectiva parental, pues no establece vínculos, un sector manifiesta sentirse solo, (Jiménez, L. 2014, p. 5). Esto trae como consecuencia un clima propenso a desarrollar conductas adictivas o problemas de socialización, Antolinez, Rivero y Torres, 2011, p.20).

En la presente tabla, se describe datos de la encuesta sobre la influencia de los estilos educativos de crianza y el uso de los dispositivos.

\section{EI PRESENTA INFLUENCIA E NO PRESENTA INFLUENCIA}

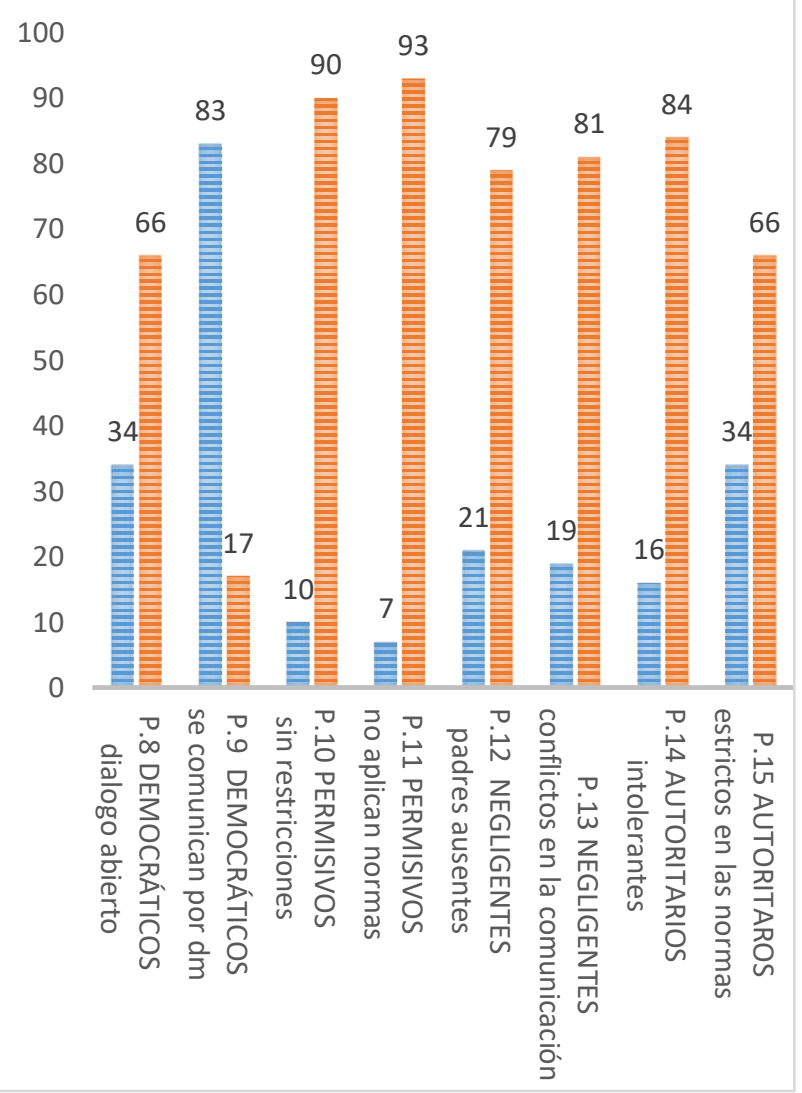

(12) Rasgos de conducta parental, de acuerdo a la clasificación de los Estilos Educativos Parentales. (Jiménez, M. 2010, p. 5 y 9). 
Se puede decir que las ventajas y desventajas encontradas en los estilos educativos de crianza, indican que en la mayoría de los encuestados existen normas de uso en el hogar (Jiménez, L. 2014, p.17), con un ligero porcentaje que no reconoce normas parentales, esto puede deberse a factores de crianza permisiva ausente, muy restrictiva o negligente. En este sentido, se puede afirmar que un gran porcentaje de los encuestados reconoce el respeto a las normas, aunque aparentemente la discusión sobre la usabilidad de los DM genere preocupación y disgustos entre los miembros, comprueba que la educación parental bien informada y abierta puede lograr establecer las reglas en casa.

\section{Resultados}

Aspectos positivos y negativos que se generan en el ámbito psicológico adolescente con el uso de los DM.

Cabe resaltar los siguientes aspectos positivos:

La usabilidad del dispositivo móvil. La comodidad del manejo de sus herramientas como de sus aplicaciones hacen de estos aparatos multiusos los mejores aliados en las relaciones interpersonales, esté presente o no en el encuentro.

D Amplia capacidad de actividades y socialización (adolescentes multitareas $y$ dispositivos multiusos). Establece que su medio de comunicación con el mundo exterior es el dispositivo móvil, el cual goza de esta característica sin límites, navega y se comunica con la simbología del lenguaje digital.

\section{> Notoriedad omnipresente con características singulares que varios investigadores denominaron como la generación}

red, generación@ o nativo digital ${ }^{13}$, conectado a través de un dispositivo móvil. Le da la oportunidad de comunicarse simultáneamente con sus contactos y obtener más información a través de las aplicaciones.

Expresión de sus estados de ánimo de forma lúdica. Afirma que el mayor pasatiempo y grado de sociabilización se da con las aplicaciones de entretenimiento más utilizadas en sus dispositivos. Los adolescentes internautas generan sus contenidos construyendo identidades lúdicas, estados de ánimo, manifestaciones de su yo, como signo emancipador del control paterno, produciendo o compartiendo flyers ${ }^{14}$, snapchats, instagram, etc.

Reafirma su espacio y concepto de sí mismo. Aplicaciones como Facebook, Whatsapp, line, Instagram o Snapchat, revelan al púber como alguien que tiene la necesidad de ser validado por sus pares y ser acogido en el grupo.

$>\quad$ Establecen sus propias reglas valorando el DM como objeto asesor de imagen, de status y moda. Por la marca más utilizada, por el diseño del hardware y la presentación del software en sus aplicaciones ${ }^{15}$. Establece sus propios criterios valorando su identidad en el círculo social que maneja.

\section{$>\quad$ Necesidad de autocontrol y autonomía. Las herramientas del DM, facilitan la organización de sus actividades de manera cronológica, reloj despertador, agenda y notas, para tareas y acontecimientos lúdicos, salidas, programas de tv., etc. esta ventaja se fundamenta con la edad crítica por la que atraviesa todo adolescente en esta etapa,}

${ }^{(13)}$ Los adolescentes actuales cumplen una característica común; han crecido en la era digital y como tales, obedecen a normas de comportamiento como la notoriedad y presencia en todos los acontecimientos que rodean su mundo virtual, lo identifican y lo valoran. (Prensky, 2010, p. 16).

(14) Volante o pieza gráfica virtual que se distribuye de manera masiva. Garrote, 2013. p. 14.

(15) Es muy frecuente la manifestación del adolescente al elegir el tipo de celular que piensa utilizar a estas edades, sobretodo que no sea de la misma marca que tenga el progenitor y mucho menos el modelo de funciones. (Enesco, 2015, p. 48.) 
desarrollando rapidez en la construcción digital, durante su proceso de emancipación. (Medina, 2013, p. 5).

Entre los aspectos negativos:

El abuso de los DM en familias conflictivas. Los encuestados confirman que mantienen varias cuentas de usuario en sus dispositivos, generando varias realidades paralelas, (Medina, 2013, p. 7) según investigadores sistémicos de la comunicación, esto puede generar manifestaciones alteradas de conducta en el adolescente, como reacciones a diversos patrones que lo afectan y el único espacio que los acoge es el celular. (Satir, 2002, p. 133).

$>$ Riesgos de dependencia de los DM. Consideran que no podrían dejar el celular en casa pues no se sienten completos. Como bien lo dijo Lorente, (2002. p. 16) "...el dispositivo móvil es la extensión del brazo adolescente...". De este modo, el adolescente manifiesta dos aspectos importantes para su desarrollo, la necesidad de comunicarse y la necesidad de emanciparse del control parental.

Admisión de falsos contactos. El grupo prefiere mantener sus relaciones sociales desde su espacio virtual, lo que puede ocasionar por la necesidad de sociabilizar dos tipos de riesgo: ser vulnerado en sus cuentas por conocidos que desean agredirlo, o buscar de otro modo "amigos que lo acepten" admitiendo contactos que no son adecuados.

Tiempo de caducidad y Obsolescencia por costo. La desventaja de estos aparatos tecnológicos es su alta caducidad en cuanto a su tiempo de uso, debido a los imparables adelantos tecnológicos. Los DM como muchos aparatos tecnológicos pierden usabilidad en menos tiempo cada vez que emergen nuevas tecnologías que los reemplacen, por lo que resulta complicado y de alto costo a los padres acceder a los nuevos dispositivos que los hijos exigen.

$>\quad$ La frecuencia de uso y la comunicación en casa. Se ha observado que hay un ligero descontrol al uso de los dispositivos en casa por un porcentaje de encuestados que podría traer consecuencias de no establecerse normas.

\section{Criterios y Normas del uso de dispositivos móviles con hijos adolescentes}

\section{Guía didáctica}

El desarrollo de la guía didáctica, está dirigida a instituciones educativas, del estado y talleres de orientación familiar, que ayuden a las familias conociendo, previniendo $y$ orientando su desarrollo en la sociedad. Para esto se elaboraron criterios recogidos del cruce de información teórica así como de antecedentes analizados, la encuesta aplicada y las opiniones de los padres de familia, llegando a determinar las normas desde fundamentos teóricos y prácticos.

El Objetivo general es orientar sobre las oportunidades y riesgos del uso de los dispositivos móviles en los adolescentes y su impacto en la comunicación familiar.

Los objetivos específicos son conocer los aspectos generales sobre los dispositivos móviles en la vida familiar, reflexionar sobre los aspectos generacionales sobre su uso, conocer fundamentos teóricos sobre la familia y tipos de comunicación familiar actuales, reflexionar sobre los retos y posibilidades que la comunicación digital ofrece a través de los dispositivos móviles y su impacto en la familia y reflexionar sobre los peligros y riesgos que estos mismos pueden generar. 
La metodología del taller es teórica y práctica, por tanto, la labor de los profesores y capacitadores es el de brindar información, compartir experiencias basadas en investigaciones científicas, así como el apoyo de la casuística actual, que motiven la interacción de los asistentes para una mejor comprensión y resultados.

\section{Conclusiones}

La comunicación y el aprendizaje empieza desde la iniciación de la vida y como tal, los padres moldean y edifican desde la concepción, todo conocimiento que complemente la experiencia que irá adquiriendo el individuo conforme se relacione con el entorno. Partiendo de este concepto, es necesario tener en cuenta que el desarrollo de la comunicación se da a partir de la concepción y de todo lo que adquiera a través cada etapa de vida.

Es así que se estableció dos grupos de reacción al estudio aplicado:

i. El primer grupo, se determinó que la mayoría de adolescentes, mantiene un adecuado uso de los dispositivos móviles en casa y en el ámbito educativo, y que además sus padres (democráticos y asertivos) manejan normas y tratan de adaptarse a los nuevos estilos comunicativos digitales de sus hijos, siendo conscientes de la influencia que ejercen las TIC, consiguen mostrar interés de acortar la brecha digital.

ii. El segundo grupo, que fue la minoría de adolescentes, presentan problemas en el uso con un abuso acrecentado de estos aparatos, debido a las dificultades de comunicación en casa, al no tener orientación o un adecuado control, sea por poco interés o demasiada restricción parental en las normas. Presentando un nivel de adicción moderado en las aplicaciones de DM.

En la caracterización de la comunicación familiar actual y el uso de los DM, se ha considerado que con la intrusión de los dispositivos móviles en la comunicación de padres e hijos adolescentes, las relaciones familiares se modifican.

Dependiendo del tipo de comunicación con el que se relacionen las familias, sea abierta o cerrada, inevitablemente se encontrarán inmersos en la expansión digital a través de estos aparatos ${ }^{16}$, influencia que de menor o mayor grado modifica las conductas de los miembros en etapa de formación. La educación parental bien informada y abierta y asertiva, puede lograr establecer las reglas en casa.

Se ha determinado que existe una manifestación sobredimensionada de algunos padres sobre el uso que hacen sus hijos de los DM, calificándolo de objeto adictivo y dependiente, cuando éstos no tienen un acercamiento normativo con sus hijos adolescentes.

De acuerdo al grado de intimidad que manifiesten padres e hijos en la relación familiar, los DM pueden ser vehículos alternativos eficaces de comunicación.

Los DM están marcando las pautas del desarrollo social en los adolescentes, en el área local $y$ en todo el mundo, porque la adolescencia en esta etapa exige la necesidad imperiosa de conectarse, jugar o crear nuevos constructos.

\footnotetext{
(16) Véase en reportes the Home BuildersInstitute. Sobre el incremento de aplicaciones para dispositivos móviles en el mundo. (25 de marzo de 2015) ubicado en http://peru21.pe/tecnologia/seis-contundentes-cifras-sobredispositivos-moviles-mundo-2215121
} 
Se ha determinado que el lenguaje más utilizado actualmente por los adolescentes de etapa temprana es el digital que, a través de dispositivos móviles, y a medida que estos se desarrollen, mayor será la necesidad de uso, pasando a ser parte fundamental de la comunicación.

Se ha observado que cuanto medio de información física o virtual se manifieste, es necesario que las familias tengan conocimiento sobre las herramientas y las aplicaciones que ellos utilicen, así como orientación sobre el peligro al que puedan estar expuestos en las redes a través del celular.

Dependiendo de cuál sea el estilo educativo que impartan a sus hijos, la concertación en un diálogo claro y asertivo constante con ellos, será la clave para evitar futuras conductas de rebeldía o negativas a la comunicación.

"Chatear" o enviase mensajes mediante dispositivos puede crear acercamientos y lazos de intimidad entre los jóvenes, pero esa conectividad, que otros autores consideran como abuso de la tecnología, radica exactamente en ese afán de estar conectado a la red todo el tiempo. El equilibrio de estos comportamientos debe ser monitoreado por los progenitores, pues son ellos los responsables de las conductas de sus hijos en etapa de formación. El orden familiar, debe ser cohesionado y adaptable pues es ahí donde las normas deben regir inalterables.

\section{Recomendaciones}

Considerando los resultados de la investigación tanto en los criterios teóricos como prácticos y las conclusiones señaladas, se plantearon las siguientes recomendaciones:
Los padres de forma asertiva pueden limitar el uso de los dispositivos en sus hijos adolescentes y pactar las horas para sus momentos de ocio.

Fomentar la socialización cara a cara; como base de la crianza educativa parental, es premisa básica para una comunicación abierta y preventiva.

> Fomentar aficiones como la lectura y actividades culturales; es un acompañamiento necesario en la crianza de los hijos.

> Fomentar actividades grupales al aire libre y servicios como el voluntariado, refuerza los valores y la convivencia sana para el desarrollo integral del adolescente.

Es importante que los padres ofrezcan a sus hijos valores pedagógicos, como el uso constructivo de internet, advirtiendo el cuidado de su intimidad, protegiendo su privacidad y evitando la publicación de información privada y fotográfica que manifieste de manera pública su intimidad.

$>\quad$ Se hace necesario que los padres se informen debidamente de todos los riesgos reales que existen con fundamentos científicos y certificados, para no caer en falsedades o distorsiones con respecto a posibles trastornos o adicciones.

Aprender a identificar los mensajes que los hijos trasmiten en sus pantallas, sin llegar a invadir sus espacios. Es importante, observar y escuchar qué es lo que manifiestan, o postean en sus muros. Investigar con quiénes se conectan regularmente y a quién siguen o quiénes los siguen. El acompañamiento y la confianza de un diálogo abierto es primordial, para evitar los problemas de Cyberbullying, siendo más comunes en jóvenes con comunicación familiar escasa o conflictiva. 
Planificar y ejecutar talleres y asesorías virtuales sobre comunicación familiar y el manejo de las TIC en dispositivos móviles. Se ha observado que en la mayoría de las investigaciones nacionales, no se han ejecutado mecanismos que regulen la orientación de uso de estos dispositivos.

Realizar un estudio de las nuevas clasificaciones de familia y la influencia que las nuevas tecnologías móviles ejercen en las relaciones comunicativas del contexto nacional.

Profundizar con instrumentos aplicados sobre los criterios teóricos y prácticos que dieron como resultado opiniones divergentes en cuanto a la usabilidad y funcionalidad de los dispositivos, analizando desde el ámbito psicológico las causas que evidencian el uso adictivo de estas tecnologías y el acoso cibernético en el contexto local.

Realizar una descripción y análisis de la simbología en la comunicación digital, sus significados y la influencian en el comportamiento de los adolescentes, desde el ámbito psicológico, ético y social.

> Implementar un sitio web o Institución para difundir contenidos que orienten el uso adecuado de los dispositivos móviles en la familia.

Realizar un estudio que analice la problemática de la comunicación digital en la etapa temprana de la infancia, proponiendo guías de orientación a los padres en el estilo educativo de crianza.

\section{Bibliografía}

Antolínez Merchán, M. y Rivero Recuenco, A.Torres Acebrón L. (2011).El Teléfono
Móvil: Usos, Normas y Conflictos Familiares, Tesis para optar el título Doctoral, España, UNAV.

\section{Enesco I. (2015.) Psicología del Desarrollo.}

Garrote Pérez de Albéniz, G. (2013), Uso y abuso de tecnologías en adolescentes y su relación con algunas variables de personalidad, estilos de crianza, consumo de alcohol y autopercepción como estudiante, Tesis para optar el grado de Doctor, España, U. de Burgos.

Herrero-Diz, P. Alcaide-Pulido, p. (2014), La comunicación digital en el aprendizaje entre iguales: niños y jóvenes como educadores en la Red. Universidad de Loyola. Andalucía, España.

Jiménez L. (2014), El WhatsApp en las prácticas de intimidad familiares, Tesis para optar el título de Maestría en la Sociedad de la Información y el Conocimiento, Universidad Oberta de Catalunya.

Jiménez, M J. (2010), Estilos educativos parentales y su implicación en los diferentes trastornos, España.

Ling, R. (2002), Telenor R\&D. "Chicas Adolescentes y Jóvenes Adultos Varones: Dos Subculturas del Teléfono Móvil", Noruega, La Revista de Estudios de Juventud, $N^{\circ} 57$, Edición $\Subset$ Instituto de la Juventud, Universidad Politécnica de Madrid.

Lorente, S. (2002), "Juventud y teléfonos móviles, algo más que una moda Revista de Estudios de Juventud. $\mathrm{N}^{\circ} 57$.

Medina Núñez, (2013), el Smartphone y la construcción de la identidad, Tesis para 
optar el título Doctoral, Pontificia Universidad Católica del Perú.

García M., Monferrer J. (2009), “Propuesta de análisis teórico sobre el uso del teléfono móvil en adolescentes", Comunicar, v. XVII, No 33.

Prensky, M. (2010) Digital Natives, Digital Inmigrants, Vol. 1, Traducido por Editorial

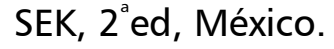

Satir, V. (2002) Nuevas Relaciones humanas en el núcleo familiar, traducido por Rodríguez y

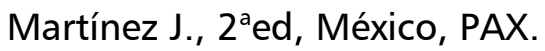

Tecnología, (4 de julio de 2015) el lado oscuro de la tecnología, Gestión recuperado de http://gestion.pe/tecnologia/lado-oscurotecnologia-2136292 